\title{
Recruitment of Patients to a Glaucoma Drug Trial in a District General Hospital
}

\author{
PALANISWAMY SUNDERRAJ and COLIN S. L. PEIRIS \\ Southport
}

\begin{abstract}
Summary
We report the difficulties experienced in identifying and recruiting 21 patients with ocular hypertension or primary open-angle galucoma to participate in a clinical trial of two topical beta-blockers. The case-notes of 374 consecutive patients expected to attend routine glaucoma clinics were reviewed to recruit 21 eligible patients who were willing to undergo the drug trial. Recruitment of patients to prospective drug trials may be complicated by a high proportion of exclusions due to ineligibility and also by refusal of otherwise eligible patients.
\end{abstract}

Recruitment of eligible subjects who are willing to take part in a clinical trial is one of the major problems encountered in clinical research. ${ }^{1,2}$ In spite of several reports from other specialties on the extent of this common problem ${ }^{2-8}$ only limited information is available in the ophthalmic literature. ${ }^{9}$ We report the difficulties experienced in identifying and recruiting patients with ocular hypertension $(\mathrm{OH})$ or primary open-angle glaucoma (POAG) to participate in a double-masked, cross-over evaluation of two different beta-blockers.

\section{Patients and methods}

The proposed, comparative, evaluation of two topical beta-blockers was to be carried out in the eye department of the District General Hospital, Southport. The study patients, already well controlled on treatment with a topical ocular hypotensive agent, were required to attend the hospital on five occasions over a period of four months with each visit lasting approximately 30 minutes. The study patients were identified and recruited in the following stages:

\section{Stage I}

The case-notes of all the patiens attending the weekly glaucoma clinic were reviewed on the preceding day to identify subjects with $\mathrm{OH}$ and POAG who fulfilled the following conditions for possible inclusion in the trialuntreated intraocular pressure greater than $21 \mathrm{~mm} \mathrm{HG}$ with or without glaucomatous visual field defect and pathological cupping of the optic disc, open angle of the anterior chamber on gonioscopy and no demonstrable secondary cause of raised intraocular pressure.

Case-notes of the patients so selected were studied in detail and excluded according to the following criteria:

(1) patients younger than 18 years or older than 80 years of age

(2) history or evidence of serious respiratory disorder including bronchial asthma

(3) history or evidence of bradycardia (less than 55 beats per minute), heart block, ischaemic heart disease or cerebro-vascular insufficiency

(4) history or evidence of neuro-psychiatric 
problems including depression and anxiety neurosis

(5) presence of chronic ocular disease like blepharitis, recurrent corneal ulceration or dry eye syndrome

(6) current contact lens wear

(7) history of glaucoma filtration surgery or intraocular surgery

(8) glaucoma controlled with more than one medication

(9) history of intolerance to beta-blocking agents

(10) taking concurrent medications with systemic beta-blockers, beta-antagonists, tri-cyclic anti-depresessants, mono-amino oxidase inhibitors, oral or topical corticosteroids, carbonic anhydrase inhibitors or other drugs which may affect intraocular pressure or interact with topical beta-blockers

(11) pregnant women or nursing mothers

(12) judged by the investigator to be unsuitable for the study

No. of notes reviewed

374

$\stackrel{\text { stage } 1: \text { rejected }}{\longrightarrow} 143(38 \%)$

No. satisfying inclusion criteria

231

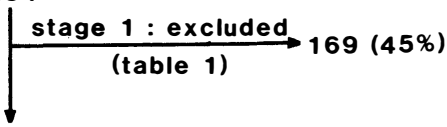

No. eligible for interview

62

stage 2 : excluded 34 (9\%)

No. willing to participate

28

$\underset{\text { after examination }}{\stackrel{\text { stage } 3: \text { excluded }}{\longrightarrow}} 4(1 \%)$

No. willing and eligible for trial

24

$\underset{\text { stage } 4: \text { no reply }}{\underset{\text { to communication }}{\longrightarrow}} 3(1 \%)$

No. eligible and formally

consented for trial

$21(6 \%)$

Fig. 1 Flow-chart summarizing the identification and recruitment of study patients.
Stage II

The patients who satisfied both the inclusion and exclusion criteria and attended the routine glaucoma clinic review were interviewed. The objectives of the study and the implications of participation were fully explained to each potential subject. Those who were unwilling to participate in the trial or were judged unsuitable were excluded from the study.

\section{Stage III}

The remainder of the potential subjects for the study underwent a comprehensive general medical examination including the cardiovascular and respiratory systems to assess their fitness for participation in the trial. A detailed medical history was also obtained. Some patients were excluded from the study at this stage due to medical problems.

\section{Stage IV}

All the willing and eligible patients were subsequently contacted to inform them that both the investigators were available for any final clarification required before the potential subjects gave formal consent for participation in the evaluation of two ophthalmic beta-blockers.

This routine is summarised as a flow-chart in Figure 1.

\section{Results}

Reveiw of the case-notes of 374 consecutive patients expected to attend the routine glaucoma clinics revealed 231 case-notes of patients with $\mathrm{OH}$ or POAG who satisfied the inclusion criteria. The remaining 143 casenotes belonged to patients with suspect glaucoma (16), narrow-angle glaucoma (34), normal tension glaucoma (16), secondary glaucoma (10) and others including postoperative and casualty patients.

One hundred and sixty-nine case-notes from the 231 initially identified for inclusion in the study were excluded due to the various causes listed in Table I. A single case-note was occasionally excluded for more than one reason. Forty-two patients were judged unsuitable for the study on the basis of information found in their case-notes (Table I) because of visual impairment (13), poor 
Table I Reasons for excluding case-notes of patients with ocular hypertension/primary open-angle glaucoma*

\begin{tabular}{|c|c|c|}
\hline Reason & Number of case notes & (percentage) \\
\hline Multiple anti-glaucoma medication & 87 & (38) \\
\hline More than 80 years old & 49 & (21) \\
\hline Judged unsuitable for the study & 42 & (18) \\
\hline Trabeculectomy & 26 & (11) \\
\hline Problems contra-indicating beta-blockers & 23 & $(10)$ \\
\hline Respiratory 17 & & \\
\hline Cardio-vascular & & \\
\hline Neuro-psychiatric & & \\
\hline Pre-existing eye disease & 11 & $(5)$ \\
\hline On prohibited medication & 10 & (4) \\
\hline Others & 21 & (9) \\
\hline
\end{tabular}

*a single case-note may have been excluded due to more than one reason

general health (12), lived or worked a long distance away (9) and other reasons (13).

Of the 62 patients who were eligible to proceed to Stage II, four failed to keep the glaucoma clinic appointment. The other patients were interviewed, of whom 13 declined to participate in the study. A further 17 patients (nervous disposition 5, poor general health 5, lived or worked a long distance away 4 , difficulty in performing appalantion tonometry 2 , other reasons 3 ) were found unsuitable for the study. The 28 remaining patients were examined in Stage III and of these, four were excluded due to cardiac (one patient) and respiratory (three patients) problems.

The 24 eligible and initially willing patients were subsequently contacted for definite participation in the study. Three patients failed to give formal consent leaving 21 patients for inclusion in the study of two topical beta-blockers.

These 21 patients were recruited from a review of the case-notes of 374 patients expected to attend routine glaucoma clinics, a ratio of $1: 18$.

\section{Discussion}

Identification and recruitment of eligible subjects who are willing to participate in a clinical trial is a major and common problem. ${ }^{1-9} \mathrm{Sev}$ eral studies from other medical specialties ${ }^{3-8}$ have reported progressive loss of patients who were initially identified as potential subjects for inclusion in the clinical trials. The ratio of final participants in the study to those who were initially identified as likely subjects varies from 1:4 in the Scottish breast conser- vation trial $^{8}$ to $1: 136$ in the Systolic Hypertension in the Edlerly Programme Study. ${ }^{7}$ In comparable ophthalmic literature Quick et al. ${ }^{9}$ reported a ratio of 1:93 (15 patients with $\mathrm{OH}$ or POAG were recruited from 1358 potential subjects for participation in a glaucoma drug trial) which is significantly different from the ratio of 1:18 experienced in the present study despite similar inclusion and exclusion criteria.

We identified potential subjects for the glaucoma drug trial by reviewing the casenotes of the patients who were to attend routine glaucoma review clinics unlike Quick et $a l .{ }^{9}$ who used an 'outpatient diagnostic index ${ }^{10}{ }^{10}$ Quick et al. ${ }^{9}$ rejected $32 \%$ of all the case-notes reviewed because these belonged to 'glaucoma suspects', a patient group which was not eligible for participation in the topical beta-blocker trial. These patients comprised only $11 \%$ of all the case-notes we reviewed because glaucoma suspects who are normally followed at yearly intervals or more, unlike glaucoma patients who are followed at shorter intervals, are more likely to be encountered in a diagnostic index system than in a regular glaucoma clinic.

The case-notes of 176 (13\% of the total) patients identified as potential subjects for the trial using the diagnostic index was not available to Quick et al. ${ }^{9}$ for the purpose of review. This problem did not arise in our study as all the case-notes except one were traced and kept ready for the routine glaucoma clinic appointment. Thus glaucoma suspects and unavailable case-notes accounted for the loss of $45 \%$ of the potential subjects identifed by 
Quick et al. ${ }^{9}$ for possible inclusion in the glaucoma drug trial compared to $11 \%$ in our study. We did not compare the frequency of other causes of exclusion of the original case-notes because the previous authors ${ }^{9}$ mention only one reason for excluding a case-note.

Twenty-two per cent (13) of the patients we invited personally to participate in our drug trial declined to do so. This was less than the $38 \%$ ( 73 patients out of the 193 invited by letter to participate in the glaucoma drug trial) reported by Quick et al. ${ }^{9}$ This may be because our study was of shorter duration, required fewer visits to the hospital and involved less of the patient's time. Possibly also, a direct personal approach by the doctor evokes patient confidence and works better than the postal approach in securing the patients' consent to undergo a clinical trial (Villada and Joyce, personal communications).

Twenty-nine per cent (17) of the interviewed patients were found ineligible to proceed to the examination stage in our study compared to the $80 \%$ ( 96 patients out of the 120 interviewed) reported elsewhere. ${ }^{9}$ The majority of the exclusions in that study ${ }^{9}$ after interview were due to patients on prohibited medication, presence of extensive field loss and cardio-respiratory disease and other reasons which would normally be found recorded in case-notes. It appears that either the case-notes did not contain all the relevant information or whilst unlikely, they were overlooked during the initial review of the case-notes. This may account for the high rate of rejections of potential subjects at this stage of recruitment. ${ }^{9}$

In summary, the progressive loss of potential subjects for participation in a glaucoma drug trial during the various stages of identification and recruitment was significantly more in the previous report ${ }^{9}$ than in our study. This was due to multiple factors like the method used to identify potential subjects for inclusion in the trial, availability of the casenotes for the subjects so identified, the design of the trial in terms of duration and number of patient visits required, the approach used in securing the patient's consent to undergo the drug trial and other miscellaneous factors. The impact of these factors on the progressive loss of patients during the organisation of any clinical trial is highly variable not only within the same medical speciality ${ }^{8,11}$ but also between different medical specialities. Hence, in our opinion, one should avoid estimating the number of subjects who can be expected finally to undergo a clinical trial by adopting a specific number such as $10^{2}$ or $100^{9}$ to divide the number of potential subjects. This may turn out to be too optimistic or conversely, dampen efforts to conduct trials in smaller District General Hospitals.

We thank Mr P. W. Joyce FRCS and Dr A. A. Adebiyi for editorial assistance, Miss J. Morton for secretarial assistance, and Mrs K. Barton and the staff of the Appointments and Medical Records Department for help in carrying out this study.

KeY Words: Beta-blocker, Identification, Clinical trial, Drug trial, Ocular hypertension, Patients, Primary open-angle glaucoma, Recruitment

\section{References}

${ }^{1}$ Hamilton M: Computer programmes for the medical man: a solution Br Med J 1965;2:1048-50.

${ }^{2}$ Ederer F: Practical problems in collaborative clinical trials Am J Epidemiol 1975;102:111-18.

${ }^{3}$ Study personnel: Recruitment of patients National Diet Heart Study: Final Report, Chapter 2 Monograph No 18 Circulation 1968;37(S1):1-40.

${ }^{4}$ Members of the Writing Committee: Hypertension detection and follow-up program co-operative group. Patient participation in a hypertension control program $\mathrm{J} A m$ Med Assn 1978;239:1507-14.

${ }^{5}$ Writing Group: Lipid research clinics program: participant recruitment to the coronary primary prevention trial. J Chron Dis 1983;36:451-65.

${ }^{6}$ Perry HM: Treatment of mild hypertension: preliminary results of a two year feasibility trial. Circ Res 1977;40(S1):1-180.

${ }^{7}$ Vogt TM, Ireland CC, Black D, Camel G, Hughes G. Recruitment of elderly volunteers for a multicenter clinical trial: the SHEP study. Controlled Clin Trials 1986;7:118-33.

${ }^{8}$ Jack WJL, Chetty U, Rodger A. Recruitment to a prospective breast conservation trial: Why are so few patients randomised? $\mathrm{Br}$ Med I 1990;301:83-5.

${ }^{9}$ Quick AM, Khaw PT, Elkington AR: Problems encountered in recruiting patients to an ophthalmic drug trial. Br J Ophthalmol 1989;73:432-4.

${ }^{10}$ Elkington AR and Davies FC: Setting up an out patient diagnostic index Proc Roy Soc Med 1980;73:646-8.

${ }^{11}$ Wilson RG, Hart A, Dawes PK: Mastectomy or conservation: the patient's choice. $\mathrm{Br}$ Med 1988;297:1167-9. 